\title{
Comparison of EMA,CEA, CD10 and Bcl-2 Biomarkers by Immunohistochemistry in Squamous Cell Carcinoma and Basal Cell Carcinoma of the Skin
}

\author{
Mazaher Ramezani ${ }^{1}$, Elham Mohamadzaheri ${ }^{1}$, Sedigheh Khazaei $^{1}$, Farid Najafi ${ }^{2}$, \\ Asad Vaisi-Raygani ${ }^{3}$, Mahtab Rahbar ${ }^{1}$, Masoud Sadeghi ${ }^{4 *}$
}

\begin{abstract}
Background: Basal and squamous cell carcinoma (BCC and SCC) are the most common skin cancers worldwide and distinction between the two may sometimes be very difficult in routine histopathology. The present study was aimed to evaluate a reliable diagnostic method for these cancers based on immunohistochemistry (IHC). Materials and Methods: IHC was used with antibodies to Bcl-2, CD10, CEA, and EMA biomarkers, which despite non-specificity are easily available for detection of various types of tumors in pathology sections and can be used as a panel for differentiation. In this descriptive and analytic study, paraffin- embedded blocks of $29 \mathrm{SCC}$ patients and 29 BCC patients were collected and sectioned for IHC staining. The results were analyzed by the STATA (version 8) statistical package using the Chi-square test. Results: BCC patients were 100\%, 75.8\%, $0 \%$ and $0 \%$ positive for Bcl-2, CD10, CEA and EMA markers, respectively, and for SCC patients were 3.5\%, $0 \%$, $34.5 \%$ and $82.7 \%$ positive, respectively. Using simultaneously Bcl-2 and CD10 as positive markers, detection of BCC with $88 \%$ accuracy and $100 \%$ specificity was possible, while application of CEA and EMA positivity could detect SCC with $67 \%$ accuracy and $100 \%$ specificity. Conclusions: SCC and BCC have different immunostaining profiles; therefore, Bcl-2, CD10, CEA and EMA markers can be helpful to distinguish between them.
\end{abstract}

Keywords: Skin cancer - BCC - SCC - differential diagnosis - immunohistochemistry - markers

Asian Pac J Cancer Prev, 17 (3), 1379-1383

\section{Introduction}

Melanoma and non-melanoma skin cancers are now the most common types of cancer in white populations (Leiter and Garbe, 2008). Among non-melanoma skin cancer, basal cell carcinoma (BCC) and squamous cell carcinoma (SCC) are the most common (Aiad and Hanout, 2007) that $80 \%$ for BCC and $20 \%$ for SCC (Halpern and Hanson, 2004). For skin cancers, it was reported as the most common cancer for both genders in Kermanshah province, Iran. In the most cases, these tumors are reported in sun-exposed areas. UV exposure is known as the main risk factor. The incidence of this type of tumors among white-skin people is on the rise (Rajabi et al., 2007). Distinguishing between BCC and SCC is clinically significant. BCC has local invasion and rarely metastasizes (according to surgical centers; its metastatic rate is $0.1 \%$ and in pathology reports is $0.01 \%$ ). BCC most commonly appears as a pearly white, dome-shaped papule with prominent telangiectatic surface vessels and SCC most commonly appears as a firm, smooth, or hyperkeratotic papule or plaque, often with central ulceration (Firnhaber, 2012). In some cases, an accurate morphological classification between SCC and BCC is both difficult and controversial (Juan Rosai,2004). Studies have been conducted on immunohistochemistry (IHC) to distinguish between BCC and SCC but with no accomplishments (Rajabi et al., 2007).

No studies have been conducted on simultaneous application of four IHC markers including; epithelial membrane antigen (EMA), carcinoembryonic antigen (CEA), cluster of differentiation 10 (CD10), and B-cell lymphoma 2 (Bcl-2) to distinguish between $\mathrm{BCC}$ and SCC; and since the above markers are also used to detect other types of tumors and are easily available, therefore; the achievements of this study could be a foundation to establish a very easy and available method to distinguish between BCC and SCC, particularly in difficult cases.

\section{Materials and Methods}

This was a descriptive and analytic study conducted

${ }^{1}$ Molecular Pathology Research Center, Emam Reza University Hospital, ${ }^{2}$ Department of Biostatistics and Epidemiology, ${ }^{3}$ Department of Clinical Biochemistry, ${ }^{4}$ Cancer Research Center, Kermanshah University of Medical Sciences, Kermanshah, Iran *For correspondence: Sadeghi_mbrc@yahoo.com 


\section{Mazaher Ramezani et al}

on the paraffin- embedded blocks of patients who had referred between 2006 and 2009 to the laboratory of Emam Khomeini Hospital, special clinic and Emam Reza hospital (Kermanshah, Iran)and were pathologically diagnosed as skin cancer including; SCC and BCC. Re-sections were implemented on all the blocks and based on H\&E staining and existing criteria, previous diagnoses of SCC and BCC were re- approved by two pathologists.

At the next stage, 4 micron sections (Minimum one section per marker) were prepared out of the aforementioned paraffin- embedded blocks and stained with the four IHC markers (CEA, EMA, CD10, Bcl-2). It is viewed by contributed pathologists that within a time equal to H\&E slide observation, EMA marker(Clone E29,N1504 DAKO, ready-to-use)indicated positive results by creating brown color in membrane and occasionally cytoplasm. A sample from meningioma tumor as positive control and a sample from tumor surrounding normal tissues as negative control were used. CD10 marker at a dilution of 1:50(M0727 DAKO) indicated positive results by creating brown color in more than $10 \%$ of tumor tissues in cytoplasm with or without membrane staining. Tonsil as positive control and oral mucosa as negative control were used and blocks with no tumor surrounding normal tissues excluded. CEA marker (Clone II-7, N1586 DAKO, ready-to-use) indicated positive results by creating focal and weak brown color in cytoplasm. Colon cancer sample as positive control and melanoma as negative control were considered. Bcl2 (Clone 124, N1587 DAKO, ready-touse)reported positive results by creating brown color in cytoplasm in more than $50 \%$ of tumor cells. Basal cells and lymphocytes that were scattered in the dermis were considered as positive control; and omission of antibody in primary stage or incubation with irrelevant antibodies were used as negative control. This study was conducted at Pathology Molecular Research Center from Emam Reza Hospital (Kermanshah, Iran).

\section{Results}

Among 58 patients, $36(62.1 \%)$ of them were male and $22(37.9 \%)$ were female with mean age of $61 \pm 17.5$. The mean age of patients with SCC was 58 vs. 63 years in BCC group ( $\mathrm{p}>0.05)$.The details are summarized in Table 1. Histological analysis of the patients indicated: 14 cases $(48 \%)$ were well differentiated SCC, $9(31 \%)$ moderately differentiated and $6(21 \%)$ poorly differentiated grade. Only one case demonstrated multifocal SCC (in ears, cheeks and forehead) while in all other cases there was a single lesion. All cases of BCC were located at face, neck and scalp except for one case which was located at the back.

\section{IHC Staining Results}

CD10 Staining CD10 focally demonstrates in skin natural basal layer and folliculosebaceous structure but is negative in endocrine glands. CD10-positive is viewed by the brown color created in cytoplasm with or without membrane staining (Figure 1). The results of CD10 marker staining in SCC and BCC, and its staining intensity are briefly presented in (Table 2). All 29 SCC patients were
CD10-negative.

It was positive in 22 out of $29(75.8 \%)$ BCC patients (Figure 1), however, its positivity in 16 out of the 22 patients was in low expression (between $10 \%-50 \%$ of the cells) while the other 6 patients $(20 \%)$ were positive in high expression (above $50 \%$ of tumor cells). A comparison between the two groups of patients with BCC and SCC exhibits a significant difference in tumor cells while CD10 marker is applied $(\mathrm{P}=0.001)$.

\section{Bcl-2 Staining}

Bcl-2 marker exhibits positive results by creating brown color in cytoplasm. Bcl-2 marker staining results in SCC and BCC, and its staining intensity are briefly presented in Table 2. Twenty-eight SCC patients (96.5\%) were Bcl-2-negative and one case in the form of 4+ (more than $75 \%$ of tumor cells) was positive.

All 29 BCC patients (100\%) were Bcl-2-positive (Figure 2$)$. Twenty patients $(68.9 \%)$ in the form of $4+$ (more than $75 \%$ of tumor cells), 6 patients $(20.6 \%)$ in the form of $3+$ (between 51-75\% tumor cells), 1 patient in the form of $2+$ (between 26-50\% tumor cells), 2 patient in the form of $1+$ (between $5-25 \%$ tumor cells) indicated

Table 1. Comparison of Clinical Features in BCC and SCC

\begin{tabular}{lccc}
\hline Variables & BCC & SCC & P-value \\
\hline Age, year & & & \\
$\quad$ Mean & 63 & 58 & $>0.05$ \\
$\quad$ Range & $(21-85)$ & $(32-85)$ & $>0.05$ \\
Sex & & & \\
$\quad$ Male & $17(58.6 \%)$ & $19(66 \%)$ & \\
$\quad$ Female & $12(41.4 \%)$ & $10(34 \%)$ & $>0.05$ \\
Tumor size, cm & & & $>0.05$ \\
$\quad$ Mean & 2 & 2.5 & \\
$\quad$ Range & $0.5-6.5$ & $0.3-6.5$ & \\
Site of tumor & & & \\
$\quad$ Face, neck and, & & & \\
scalp & $28(96.5 \%)$ & $26(89 \%)$ & \\
Limbs & 0 & $3(11 \%)$ & \\
Back & $1(3.5 \%)$ & 0 & \\
\hline \multicolumn{2}{l}{ BCC, Basal Cell Carcinoma; SCC, and squamous cell carcinoma }
\end{tabular}

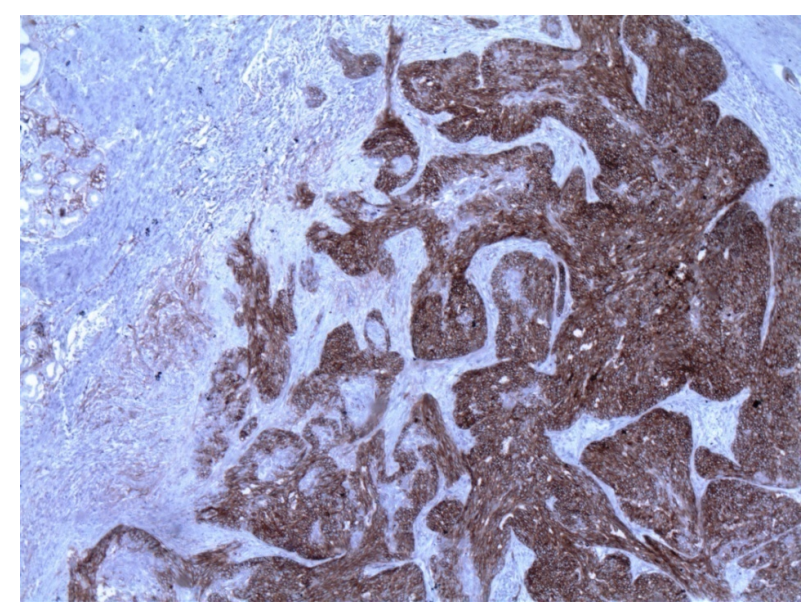

Figure 1. Immunoreactivity for CD10 in Basal Cell Carcinoma (X100) 

Table 2. Biomarkers Staining in BCC and SCC

\begin{tabular}{|c|c|c|c|}
\hline Biomarker staining, $\mathrm{n}(\%)$ & SCC & $\mathrm{BCC}$ & $\mathrm{P}$ \\
\hline CD10 & & & 0.001 \\
\hline Negative $(0-9 \%)$ & $29(100)$ & $7(25)$ & \\
\hline Low expression $(10-50 \%)$ & 0 & $16(55)$ & \\
\hline High expression $(>50 \%)$ & 0 & $6(20)$ & \\
\hline $\mathrm{Bcl}-2$ & & & 0.001 \\
\hline $0(<5 \%)$ & $28(96.5)$ & 0 & \\
\hline $1+(5-25 \%)$ & 0 & $2(6.9)$ & \\
\hline $2+(26-50 \%)$ & 0 & $1(3.6)$ & \\
\hline $3+(51-75 \%)$ & 0 & $6(20.6)$ & \\
\hline $4+(>75 \%)$ & $1(3.5)$ & $20(68.9)$ & \\
\hline CEA & & & 0.001 \\
\hline Negative (0) & $19(65.5)$ & $29(100)$ & \\
\hline Weak focal $(1+)$ & $7(24.1)$ & 0 & \\
\hline Intermediate intensity $(2+)$ & $2(6.9)$ & 0 & \\
\hline Dense staining in majority of cytoplasm(3+) & $1(3.4)$ & 0 & \\
\hline EMA & & & 0.001 \\
\hline Positive & $24(82.7)$ & 0 & \\
\hline Negative & $5(17.3)$ & $29(100)$ & \\
\hline
\end{tabular}

BCC, Basal Cell Carcinoma; SCC, and squamous cell carcinoma, EMA, epithelial membrane antigen; CEA, carcinoembryonic antigen; CD10, cluster of differentiation 10; Bcl-2, B-cell lymphoma 2 .

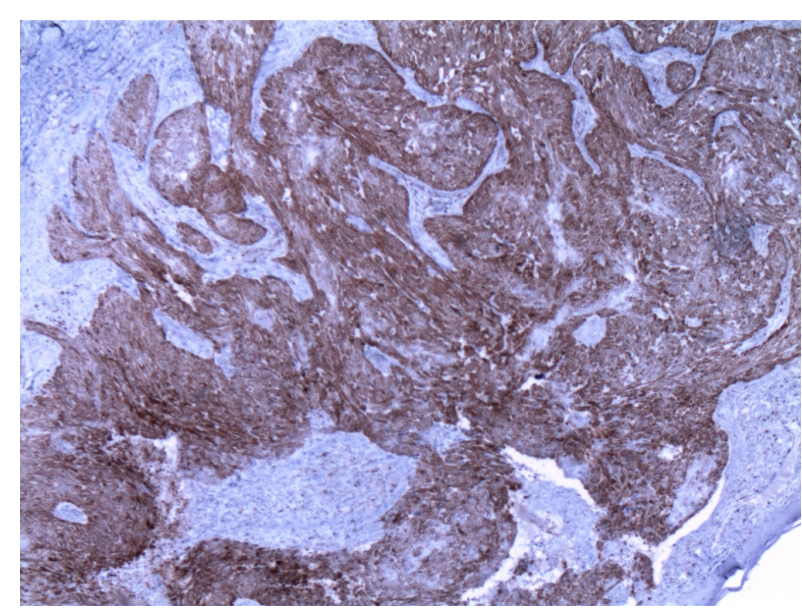

Figure 2. Immunoreactivity for Bcl-2 in Basal Cell Carcinoma (X100)

positive results. A comparison between the two groups of BCC and SCC patients exhibits a significant difference in tumor cells, while Bcl-2 marker is applied $(\mathrm{P}=0.001)$.

\section{CEA Staining}

CEA marker indicated positive results by creating focal and weak brown color in cytoplasm. CEA marker staining results in SCC and BCC, and its staining intensity are briefly presented in (Table 2). All BCC patients (100\%) were CEA-negative. Nineteen out of 29 SCC patients (65.5\%) exhibited CEA-negative and the other 10 patients (34.6\%) were CEA-positive. Seven Out of these 10 patients (24.1\%)in the form of $1+$, and 2 patients $(6.9 \%)$ in the form of $2+$, and 1 patient (3.4\%) in the form of $3+$ exhibit positive results (Figure 3 ).

A comparison between the two groups of patients with $\mathrm{BCC}$ and SCC exhibits a significant difference in tumor cells while CEA marker is applied $(\mathrm{P}=0.001)$.

\section{EMA Staining}

EMA marker demonstrates positive results by creating

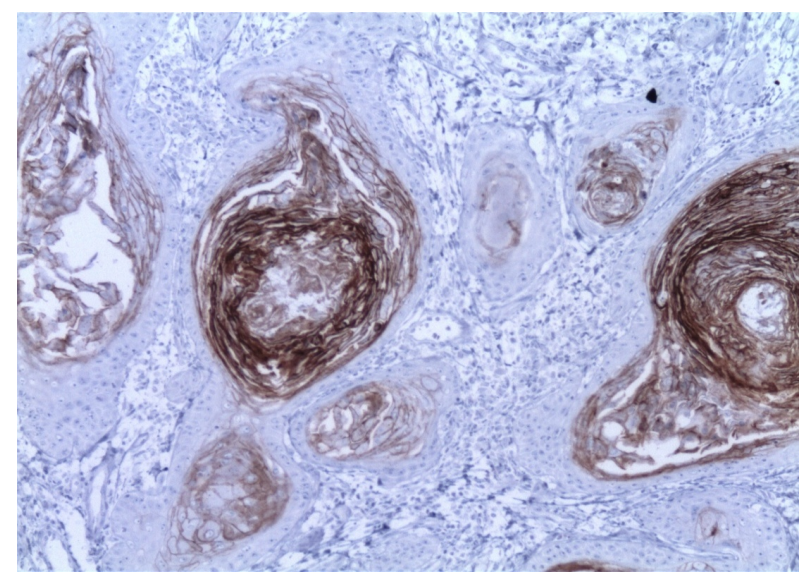

Figure 3. Immunoreactivity for CEA in Squamous Cell Carcinoma (X100)

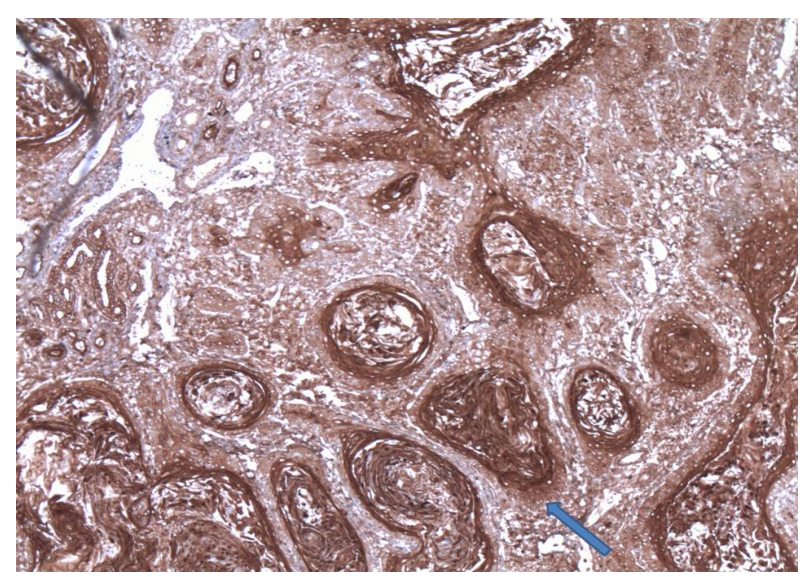

Figure 4. Immunoreactivity for EMA in Squamous Cell Carcinoma (X100)

brown color in a form of membrane and occasionally cytoplasm without staining the background tissue. All BCC patients (100\%) were EMA-negative. Twenty four out of 29 SCC patients (82.7\%) were EMA-positive 


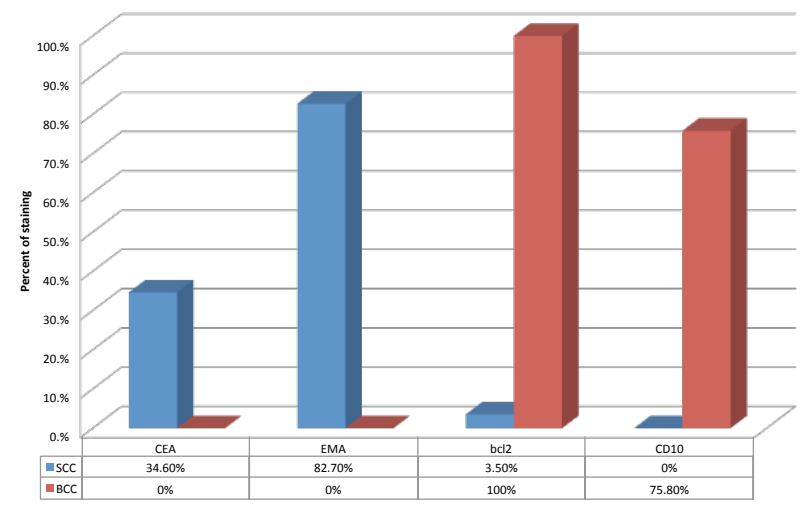

Figure 5. Biomarkers Staining Results in Basal Cell Carcinoma and Squamous Cell Carcinoma

(Figure 4), and the other 5 patients (17.3\%) were negative. A comparison between the two groups of patients with BCC and SCC showed a significant difference in tumor cells while EMA marker is applied $(\mathrm{P}=0.001)$. The results of EMA marker staining is briefly presented in (Table 2).

Regarding the higher incidence of Bcl-2 and CD10 in BCC (Figure 5), simultaneous application of both markers to distinguish BCC and SCC was taken into consideration. Twenty BCC patients simultaneously indicated both markers, while simultaneous incidence of the two markers wasn't observed in any SCC patient. Based on the above, once Bcl-2 and CD10 markers simultaneously indicate positive results then sensitivity, specificity, and accuracy of the mentioned panel to detect BCC can be calculated as following; Sensitivity: $76 \%$ Specificity: $100 \%$ Accuracy: $88 \%$.

Regarding the higher incidence of CEA and EMA in $\mathrm{SCC}$, it is taken into consideration to apply both markers simultaneously to distinguish SCC and BCC. Ten SCC patients indicated both markers simultaneously while simultaneous incidence of both markers wasn't indicated in any BCC patient. Based on the above, when CEA and EMA markers simultaneously indicate positive results then sensitivity, specificity, and accuracy of the mentioned panel to detect SCC can be calculated as following; Sensitivity: 34\% Specificity: 100\% Accuracy: 67\%

\section{Discussion}

Basal and squamous cell carcinoma (BCC and SCC) are the two most common skin cancers among Caucasian or white-skinned populations (Aiad and Hanout,2007). A distinguish between these two tumors is significantly important because $\mathrm{BCC}$ rarely metastasizes and is treatable by radiotherapy but SCC has a higher risk of metastasis and radiotherapy is not a sufficient treatment for it. These two tumors could be easily distinguished by applying morphologic criteria (Rajabi et al., 2007). However, in some cases it becomes very difficult to distinguish them particularly when a sample is small or injured. In such cases application of adjunctive techniques such as IHC is inevitable (Aiad and Hanout,2007; Rajabi et al., 2007). In this study, IHC staining with CD10, BCL-2, EMA, and CEA markers were applied on these tumors and the results were analyzed and compared with similar studies.
No patient with SCC indicated CD10 marker which is in consistency with previous studies. Twenty-two out of 29 BCC patients (75.8\%) were CD10-positive which is consistent with the findings of the previous studies (47.6\%-87.5\%) (Yada et al., 2004; Wagoner et al., 2007).

Furthermore, epidermis basal layer, inner root sheath, hair matrix, and fibrous sheath around hair follicles are CD10-positive, on the other hand CD10 staining in BCC peripheral area was more intense than its center which was similar to a pattern that exist in other adnexal tumors. However CD10 is used as a marker in the IHC panel for distinguishing trichoepithelioma and basal cell carcinoma (Tebcherani et al., 2012).These findings support the theory that $\mathrm{BCC}$ is originated from folliculo-sebaceous apocrine unit (Aiad and Hanout,2007; Yada et al., 2004), furthermore; can reflect the fact that BCC and SCC are biologically different (Aiad and Hanout,2007).

According to literature all $\mathrm{BCC}$ patients were Bcl-2positive while SCC shows negativity for this marker (83$97 \%$ of patients with negative results) (Morales-Ducret et al., 1995; Verhaegh et al., 1995; Niu et al., 2002; Lo Muzio et al., 2003; Zheng et al., 2005; Radic et al., 2011; Tebcherani et al., 2012). Our findings were similar to these studies but were in contrast to the study that found $67 \%$ and $100 \%$ positivity for Bcl-2 marker in their BCC and SCC patients, respectively (Nakagawa et al., 1994). It is possible that this Bcl-2 positivity in SCC patients might be due to use of different antibodies or different technique (Puizina-Ivic et al., 2008).

All BCC patients were EMA-negative while 24 out of 29 SCC patients $(82.7 \%$ ) were EMA-positive which shows consistency with other studies in literature review with EMA positivity between 78.5\%-95.6\% in SCC and 0-6.3\% in BCC patients (Heyderman et al., 1984; Sinard,1999; Beer et al., 2000; Rajabi et al., 2007; Sramek et al., 2008; Ansai et al., 2011; Alhumaidi et al., 2012).

All BCC patients in our study were CEA-negative. Similar studies show CEA negativity between 90 and $100 \%$ (Heyderman et al., 1984; Ansai et al., 2011) however our SCC patients show $45.5 \%$ positivity using CEA marker. This was $80-100 \%$ in other studies (Scurry and de Boer,1983; Ansai et al., 2011).

In conclusions, Findings of the present study demonstrated high efficiency of CEA, EMA, Bcl-2, CD10 biomarkers in distinguishing between SCC and BCC. Furthermore, regarding the different IHC staining profiles of these and since SCC and BCC have different IHC staining profile, application of these markers will be very helpful in such cases that routine microscopy can't distinguish between these two tumors.

\section{Acknowledgements}

The authors gratefully acknowledge the Research Council of Kermanshah University of Medical Sciences (Grant Number: 89009) for the financial support.

This work was performed in partial fulfillment of the requirements for (Residency of Pathology) of (Elham Mohamadzaheri), in Faculty of Medicine. Kermanshah University of Medical Sciences, Kermanshah, Iran. 


\section{References}

Aiad HA, Hanout HM (2007). Immunohistochemical expression of CD10 in basal and squamous cell carcinomas. J Egypt Natl Cancer Inst, 19, 195-201.

Alhumaidi A (2012). Practical immunohistochemistry of epithelial skin tumor. Indian J Dermatol Venereol Leprol, 7, 698-708.

Ansai S, Takeichi H, Arase S, et al (2011). Sebaceous carcinoma: an Immunohistochemical reappraisal. Am J Dermatopatho, 33, 579-87.

Beer TW, Shepherd P, Theaker JM (2000). Ber EP4 and epithelial membrane antigen aid distiniction of basal cell, squamous cell and basosquamous carcinoma of the skin. Histopathol, 37, 218-23.

Firnhaber JM (2012). Diagnosis and treatment of basal cell and squamous cell carcinoma. Am Fam Physician, 86, 161-8.

Halpern AC, Hanson LJ (2004). Awareness of, knowledge of and attitudes to nonmelanoma skin cancer (NMSC) and actinic keratosis (AK) among physicians. Int J Dermatol, 43, 638-42.

Heyderman E, Graham RM, Chapman DV, et al (1984). Epithelial markers in primary skin cancer: an immunoperoxidase study of the distribution of epithelial membrane antigen(EMA) and carcinoembryonic antigen (CEA) in 65 primary skin carcinomas. Histopathol, 8, 423-34.

Juan Rosai (2004). Skin. In: Juan Rosai, editor. Rosai and Ackermans surgical pathology, $9^{\text {th }}$ edition, mosby, 133-138.

Leiter U, Garbe C (2008). Epidemiology of melanoma and nonmelanoma skin cancer--the role of sunlight. $A d v \operatorname{Exp}$ Med Biol, 624, 89-103.

Lo Muzio L, Mignogna MD, Pannone G, et al (2003). Expression of bcl-2 in oral squamous cell carcinoma: an immunohistochemical study of 90 cases with clinicopathological correlations. Oncol Rep, 10, 285-91.

Morales-Ducret CR ,Van de Rijn M, LeBrun DP, et al(1995). Bcl-2 expression in primary malignancies of the skin. Arch Dermatol, 131, 909-12.

Nakagawa K, Yamamura K, Maeda S, et al (1994). Bcl-2 expression in epidermal keratinocyticdiseases. Cancer, $\mathbf{7 4}$, 1720-4.

Niu Y, Zhou Z, Liu F, et al (2002). Expression of P16 and Bcl2 protein in malignant eyelid tumors. Chin Med J (Engl), 115, 21-5.

Puizina-Ivic N, Sapunar D, Marasovic D, et al (2008). An overview of Bcl-2 expression in histopathological variants of basal cell carcinoma, squamous cell carcinoma, actinic keratosis and seborrheickeratosis. Coll Antropol, 32, 61-65.

Radic J, Batinac T, Hadzisejdic I, et al (2011). Concurrent basal cell and squamous cell carcinomas associated with hydroxyureatherapy. Acta Dermatovenerol Croat, 19, 183-6.

Rajabi P, Aboutalebdokht M, Heidarpour M, et al (2007). Evaluation of diagnostic values of EMA and Ber-Ep4 in distinction between basal cell carcinoma and squamous cell carcinoma of the skin. Iranian J Pathol, 2, 7-10.

Scurry J, de Boer WG (1983). carcinoembryonic antigen in skin and related tumors as determined by immunohistological techniques. Pathol, 15, 379-84.

Sinard JH (1999). Immunohistochemical distinction of ocular sebaceous carcinoma from basal cell and squamous cell carcinoma. Archophthalmol, 117, 776-83.

Sramek B, Lisle A, Loy T (2008). Immunohistochemistry in ocular carcinomas. J Cutan Pathol, 35, 641-6.

Tebcherani AJ, de Andrade HF Jr, Sotto MN (2012). Diagnostic utility of immunohistochemistry in distinguishing trichoepithelioma and basal cell carcinoma: evalution using tissue microarray samples. Modpathol, 25, 1345-53.
Verhaegh ME, Sanders CJ, Arends JW, et al (1995). Expression of the apoptosis-suppressing protein Bcl-2 in non-melanoma skin cancer. Br J Dermatol, 132, 740-4.

Wagoner J, Keehn C, Morgan M (2007). CD10 Immunostaining differentiates superficial basal cell carcinoma from cutaneous squamous cell carcinoma. Am J Dermatopathol, 29, 555-58.

Yada K, Kashima K, Daa T, et al (2004). Expression of CD10 in basal cell carcinoma. Am J Dermathopathol, 26, 463-71.

Zheng Z, Kye Y, Zhang X, et al (2005). Expression of p63, Bcl2 and P16 in basal cell carcinoma and squamous cell carcinoma of the skin. Korean J of Pathol, 39, 91-8. 